\title{
Making our nursing research matter
}

\author{
Sally Thorne \\ 'University of British Columbia, Faculty of Applied Science, School of Nursing. British Columbia, Canada.
}

How to cite this article:

Thorne S. Making our nursing research matter. Rev Bras Enferm [Internet]. 2016;69(5):763-4. DOI: http://dx.doi.org/10.1590/0034-7167-2015-0156

Nurses worldwide are being increasingly exposed to new and interesting research methodologies. At the same time, they are feeling considerable pressure to ensure that the studies they propose are sufficiently methodologically rigorous to pass the scrutiny of review for funding or publication. In this context, it is increasingly important to remind ourselves that methodological rigor does not necessarily produce relevance. As members of a health care profession that takes great pride in the covenant of our special social contract, we need to repeatedly remind ourselves that the purpose of nursing research is not simply to do it, but rather to use it in the service of our mission on behalf of society.

In the conventional scientific tradition, it is common to rely heavily on objective methodological criteria to assess the caliber of a proposed or completed study. We evaluate pieces of research in accordance with a predetermined set of criteria developed to permit a measure of reliability associated with the interpretation of such aspects as sample size, data collection procedures, use of theorizing, and overt expression of limitations. We understand that these criteria have evolved from an appreciation for the ways in which faulty logic can creep into a study design, rendering its findings useless or misrepresentative. However, it is also important to remember that, for the most part, the study designs we draw from have been handed down to us from disciplines whose focus of inquiry was far less complex and "messy" than the kinds of embedded and dynamic human problems about which nursing tends to be concerned. In the actual practice of our research, isolating aspects of human experience from their natural context, limiting our selection to identifiably typical cases, controlling for variation, and many other familiar aspects of conventional methodological tradition may actually weaken our capacity to do justice to the ideas that are most clearly relevant to the knowledge nursing needs to enact its core purpose and fulfil its societal mandate.

While I expect we would all agree that attention to method is fundamental to designing a study such that its findings will be credible to those who will read them, I hope we can also agree upon a comparable enthusiasm for ensuring study design does not become the central driver of what we do. If we care more about design than relevance, our attention inevitably becomes oriented toward narrow problems, those in which there are dimensions that can be removed from their dynamic human context, measured and quantified, generalized and reduced to selected variables. If instead we retain a passion for relevance, then we must force our methods to do justice to the kinds of questions about which nursing is actually deeply concerned.

The way nurses conceptualize the complex problems of health and health care in an evolving world rarely lends itself to tightly constrained study designs. For example, while we can appreciate the value of understanding diseases as biological problems, and we do know a lot about bodies, our core practice focusses our attention not directly on the narrow issue of disease entities but rather on the broader scope of human beings - in all of their social, emotional and contextual complexities - who are struggling to live their lives with those diseases. Further, while we are naturally interested in understanding common patterns of health and illness experience as relevant to our capacity to anticipate need and coordinate an informed response, we are also highly attuned to the notion of "individual difference" so that our general knowledge can be applicable to the infinite range of human variation that we expect to encounter in actual nursing practice. We nurses therefore engage with knowledge in a style of praxis that allows us to move iteratively between that which is general across populations 
and that which is a particular application relevant to each individual case ${ }^{(1)}$. Because of this, decontextualized information offers little utility within our collective disciplinary knowledge development.

I believe that it is this sincere desire to put research to good use in fulfilling our profession's larger aspirations that has led so many nurses to modify conventional methodologies, to advance options in mixed method approaches and to generate unconventional methodological applications $\mathrm{s}^{(2)}$. In many instances nurses have led the way in articulating the logic of new study protocols and forms of research design that push the boundaries of convention toward the exciting domain of trying to struggle with difficult issues as they present themselves to us in the real world. For nurses, the essence of the social contract is to keep trying to engage with the world in ways that will make a difference.

As we continue to expand upon and develop technique beyond the conventional research approaches of our cousins in the natural and social science research family, I envision a world in which nursing scholars will be truly celebrated for the creativity of their inquiry approaches and the intelligence of their study design modifications. Toward this end, we need to educate a new generation of nurse scholars with not only the capacity to fluently speak the language of conventional research methodology, but also the confidence and proficiency to lead an ongoing dialogue about when and how to break down the walls of convention. It will be this new generation that ensures we have the tools and methods to study things that really matter in ways that best represent the kinds of knowledge needed to inform excellent nursing and health care practice.

\section{REFERENCES}

1. Thorne S, Sawatzky R. Particularizing the general: Sustaining theoretical integrity in the context of an evidencebased practice agenda. ANS Adv Nurs Sci [Internet]. 2014[cited 2015 Nov 16];27(1):5-18. Available from: http:// www.ncbi.nlm.nih.gov/pubmed/24317007

2. Thorne S. Applied interpretive approaches. In: Leavy P, editor. The Oxford handbook of qualitative research. New York: Oxford University Press; 2014. p. 99-115. 